\title{
VALOR NUTRICIONAL DEL PAN DE SAL TIPO ROLLO ELABORADO CON \\ BIENESTARINA MAS ${ }^{\circledR}$
}

\section{NUTRITIONAL VALUE OF SALT PAN TYPE ROLL PREPARED WITH BIENESTARINA MAS ${ }^{\circledR}$}

\author{
*Botia R. Irene Y., Cardona A. Gabriel \\ Universidad de Pamplona, Facultad de Salud. Departamento de Nutrición. Kilómetro 1 vía Bucaramanga \\ Correo electrónico: *irenebotia@gmail.com
}

Recibido 29 de Julio 2015; aceptado 30 de octubre de 2015

\section{RESUMEN}

Colombia continúa enfrentando problemas de desnutrición y anemia por deficiencia de hierro en la población infantil. Sin embargo, se ha avanzado, realizando grandes esfuerzos para combatirlos, implementando políticas y programas de asistencia alimentaria y nutricional para la primera infancia liderados por el Instituto Colombiano de Bienestar Familiar (ICBF), en los cuales la Bienestarina Mas ${ }^{\circledR}$ como Alimento Complementario Fortificado (ACF) permite cerrar las brechas de nutrientes que existen en sus dietas habituales, mejorando el aporte de nutrientes y energía, aun cuando actualmente no se cumple con la recomendación de ingesta diaria para este grupo poblacional. Al incluir este (ACF) como parte de la materia prima en la elaboración de pan, se mejora notablemente la calidad nutricional de éste producto. El objetivo del presente trabajo fue determinar el aporte nutricional del pan elaborado con sustitución parcial de harina de trigo fortificada en $30 \%, 40 \%$ y $50 \%$. A los panes elaborados se les realizaron análisis por triplicado de proteínas, carbohidratos, grasa y fibra. Los resultados 
Autor a quien dirigirse la correspondencia. *Botia R. Irene Y. Correo electrónico: irenebotia@gmail.com obtenidos mostraron que se presentó un incremento significativo y progresivo en el contenido proteíco (9.74\%$12,63 \%)$ y en el contenido de fibra (25,7 \%- 26,90\%), convirtiéndose este producto en una excelente alternativa para variar la forma de suministro de este alimento y mejorar la calidad en la alimentación de la población infantil vulnerable de Colombia.

Palabras Bienestarina mas $\AA$, fibra, pan, proteína.

\section{ABSTRACT}

Colombia continues to face problems of malnutrition and iron deficiency anemia in children. However, progress has been made, making great efforts to fight them, implementing policies and programs for food and nutrition assistance for early childhood led by the Colombian Family Welfare Institute (ICBF), in which the Bienestarina More ${ }^{\circledR}$ as FCF allows to close the breaches of nutrients that exist in their regular diets and improves the supply of nutrients and energy, although it is not currently meet the recommended daily intake for this population group. inclusion the fortified complementary food (FCF) as part of the raw material in making bread, greatly improves the nutritional quality of this product. The aim of this study was to determine the nutritional value of bread made with partial substitution of wheat flour fortified wheat by $30 \%$, $40 \%$ and $50 \%$. A processed breads were performed in triplicate analysis of proteins, carbohydrates, fat and fiber. The results showed that there was a significant and progressive increase in protein content (9.74\% -12.63\%) and fiber content (25.7\% - $26.90 \%)$, making this product an excellent alternative to vary the shape of the food supply and improve food quality in the vulnerable children of Colombia.

Keywords: Bienestarina Mas ${ }^{\circledR}$, fiber, bread, protein. 


\section{INTRODUCCIÓN}

Las experiencias con los (ACF) se han realizado como parte de programas públicos generales subsidiados por los gobiernos de los Sectores de Salud y Bienestar Social a través de los Programas Nacionales de Alimentos Complementarios o los Programas Nacionales de Seguridad de los Alimentos (UNICEF, 2006). En Colombia la Bienestarina Mas ${ }^{\circledR}$ es el ACF suministrado sin costo alguno por el ICBF a la población vulnerable beneficiaria de los programas de asistencia alimentaria. Este complemento en su nueva formulación contiene una mezcla de cereales, leguminosas y leche en polvo, con vitaminas, minerales (Hierro, Zinc, Cobre) y Ácidos Grasos esenciales (Omega 3) (ICBF, 2014).

Este complemento alimentario puede ser utilizado en la elaboración de diferentes productos con el fin de mejorar el aporte nutricional siendo una de las alternativas para incrementar su consumo su utilización en la elaboración de pan; el cual es un producto alimenticio resultante de la fermentación y horneo de una mezcla básica de harina de trigo, agua, levadura y sal, que puede contener otros ingredientes $\mathrm{y} / \mathrm{o}$ aditivos permitidos por la legislación vigente (NTC 1363).
Nutricionalmente es un alimento rico en almidón e hidratos de carbono, esenciales por su aporte energético. (Gil, 2010). Cuando se emplean harinas de otros cereales, el pan se designa con el apelativo correspondiente a la clase de cereal que se utilice (por ejemplo, pan de centeno, de maíz entre otros), (Ávila et al., 2007).

Se ha buscado alternativas de implementación de otros tipos de harinas para la industria panadera; de hecho, se ha realizado investigaciones para ayudar a resolver esta situación en donde se sustituye parcialmente la harina de trigo por las de yuca, arroz, maíz, sorgo y otros, y se ha demostrado que, desde el punto de vista tecnológico, se puede producir pan con estas mezclas de harinas y obtener características comparables a las del pan con $100 \%$ harina de trigo (Henao et al., 2009).

Para Calaveras (1996), los cereales y sus productos derivados como el pan cubren alrededor de $40 \%$ del aporte energético del mundo. 


\section{MATERIALES Y MÉTODOS}

\section{Materiales}

Pan de sal tipo rollo elaborado con Bienestarina Mas ${ }^{\circledR}$.

\section{Métodos}

Al producto final es decir los panes se les realizaron la respectiva caracterización bromatológica para conocer su porcentaje de: grasa, carbohidratos, proteínas, calorías, y fibra cruda; siguiendo los métodos internacionales de la AOAC, métodos oficiales de análisis de alimentos.

\section{Determinación de proteínas}

Se empleó el equipo Kjeldahl, Digestión $\mathrm{K}:-425$, y Destilación K-355, se analizaron por duplicado $0,5 \mathrm{~g}$ de muestra de pan junto con $10 \mathrm{~g}$ de sulfato potásico, $0,5 \mathrm{~g}$ de sulfato cúprico y $20 \mathrm{ml}$ de ácido sulfúrico concentrado (98\%) en tubos de digestión previamente identificados.

Una vez terminada la etapa los tubos fueron retirados del digestor para esperar que disminuyera la temperatura y así poder adicionar $20 \mathrm{ml}$ de agua destilada. Para la etapa de destilación, el equipo contuvo un depósito con agua destilada, otro para ácido bórico al $2 \%$ p/v y otro para hidróxido de sodio al $30 \%$, el tubo con la muestra se colocó en el lugar de destilación con 3 gotas de indicador rojo de metilo. Este $\mathrm{NH}_{3}$ es retenido en una solución; y la cantidad de nitrógeno amónico en esta solución de $\mathrm{HCL}$ $0,1 \mathrm{~N}$ es valorada. El contenido de proteína se calculó multiplicando por factor 6.25 , estimando una proporción entre la proteína y el nitrógeno (A.O.A.C 920.87 18th edición).

\section{Determinación de grasa}

Usando un equipo Soxlhet (P-Selecta-DetGrass) se extrajeron las grasas de modo semi continuo, con un disolvente orgánico. Se calentó y se volatilizó el disolvente; este se condensó por encima de la muestra. El disolvente goteo sobre la muestra y la empapo, para extraer las grasas. A intervalos de 15 - 20 minutos, se sifonó el disolvente hasta el matraz de ebullición para empezar de nuevo el proceso. contenido de grasa se midió por la pérdida de peso de la muestra, o por el peso de la grasa extraída. (A.O.A.C. 7.060/84).

\section{Determinación de Carbohidratos.}

El extracto libre de nitrógeno (ENN) mide el contenido de carbohidratos no estructurales presentes en el contenido celular, estos son monosacáridos, disacáridos, trisacáridos y almidones. El extracto libre de nitrógenos se midió 
siguiendo el procedimiento establecido en la Guía de Análisis Bromatológico de los alimentos - Ciencias Bromatológicas de la Universidad de Pamplona a través del siguiente cálculo matemático:

Extracto no nitrogenado $=100 \%-(\%$ Humedad $+\%$ de grasa $+\%$ de fibra $+\%$ de proteína) Rojas, (2009).

\section{Determinación de fibra}

En el equipo de fibra con sistema de calentamiento $E \& Q$ se sometió la muestra seca y desengrasada a una primera digestión ácida y posteriormente a una segunda alcalina, tratamiento secuencial con ácido y base. La materia orgánica del residuo obtenido se consideró la fibra cruda. (A.O.A.C. 7.066/84 y 962.09/90).

\section{Valor calórico}

El contenido energético de un alimento se expresa en unidades de medidas llamadas Calorías cuando estos nutrientes son utilizados por el organismo 1 gramo de grasa aporta 9 calorías, mientras que 1 gramo de carbohidratos y proteínas aportan 4 calorías respectivamente. La fórmula utilizada fue la siguiente:

Calorías: ( $\mathrm{g}$ de proteínas $+\mathrm{g}$ de carbohidratos $) \times 4+($ g de grasa $\times 9)$.

\section{RESULTADOS Y DISCUSIÓN}

Los resultados correspondientes al aporte nutricional de los panes elaborados con y sin Bienestarina Mas $\AA$ se presentan en la tabla 1.

Tabla 1. Contenido nutricional del pan elaborado con y sin Bienestarina Mas ${ }^{\circledR}$

\begin{tabular}{|c|c|c|c|c|c|}
\hline \multirow[t]{2}{*}{ Variable } & \multirow{2}{*}{$\begin{array}{l}\text { Unidad } \\
\text { de } \\
\text { medida }\end{array}$} & \multicolumn{4}{|c|}{ Sustitución } \\
\hline & & $\begin{array}{c}\text { Testi } \\
\text { go }\end{array}$ & $0 \%$ & $40 \%$ & $50 \%$ \\
\hline Proteína & $g / 100$ & $9,74_{a}$ & $\begin{array}{c}11,7 \\
2 b\end{array}$ & $\begin{array}{c}12,0 \\
3 \mathrm{c}\end{array}$ & $\begin{array}{c}12,6 \\
3 d_{d}\end{array}$ \\
\hline Grasa & $\%$ & $4,57_{\mathrm{a}}$ & 4,28 & 4,00 & 3,54 \\
\hline $\begin{array}{l}\text { Carbohi } \\
\text { dratos }\end{array}$ & $\%$ & & $\begin{array}{c}28,9 \\
5 b\end{array}$ & $\begin{array}{c}29,1 \\
2 \mathrm{a}\end{array}$ & $\begin{array}{c}31,7 \\
4{ }_{a}\end{array}$ \\
\hline
\end{tabular}

Media $n=3$. Valores seguidos de la misma letra en la misma fila No son significativamente diferentes a nivel de significancia del $5 \%$.

El contenido proteico presentó un aumento significativo y progresivo, proporcional al aumento de la sustitución de Bienestarina Mas ${ }^{\circledR}$ por harina de trigo fortificada $(9,74$ \% - 12,63 \%). La sustitución del 50 \% aumentó el contenido proteico en un $30 \%$, representado en su contenido de leche y soya respecto a la harina de trigo; se han reportado estudios con incrementos de 
proteína del 4 al 15\% (Sanz, et al., 2011, Pascual et al., 2010). Valores inferiores a los reportados en esta investigación.

En cuanto al contenido de grasa se observa una disminución significativa en las sustituciones del $30 \%$ y $50 \%(4,28 \%$, $3,54 \%$ sin embargo el contenido de grasa para los diferentes productos elaborados está muy por debajo de las recomendaciones para el contenido nutricional de ACF (12,7\%), lo cual podría tener implicaciones para el estado nutricional de los niños, debido al rol critico de las grasas esenciales para el crecimiento y desarrollo de los niños, particularmente los primeros años de vida (Uauy, 2003).

El porcentaje de carbohidratos que presentó una disminución significativa correspondió a la sustitución del 30\%, debido a su alto contenido de fibra $y$ humedad las demás no presentaron diferencias significativas ya que el $p$ valor es de 0,018 y $p<0,05$; los valores de este macronutriente están por debajo a los reportado por Reyes et al., (2004) (51,55 \%) y León et al., (2011) (55,13\%).

En cuanto al contenido de fibra los resultados se presentan en la tabla 2 .

Tabla 2. Contenido de fibra en pan elaborado con y sin Bienestarina Mas ${ }^{\circledR}$

\begin{tabular}{|c|r|r|r|c|}
\hline \multirow{2}{*}{ Formulaciones } & \multicolumn{4}{|c|}{ Contenido de fibra } \\
\cline { 2 - 5 } & \multicolumn{1}{|c|}{ Mínimo } & Máximo & Media & Desviación estándar \\
\hline Testigo & 25,80 & 26,10 & 25,95 &, 15 \\
$30 \%$ & 26,07 & 26,80 & 26,36 &, 39 \\
$40 \%$ & 26,20 & 27,40 & 26,90 &, 62 \\
$50 \%$ & 23,50 & 26,40 & 25,37 & 1,62 \\
\hline
\end{tabular}

Fuente: Autor 2014

La variable de fibra no cumple los supuestos para aplicar prueba ANOVA por lo que se aplicaron las pruebas de estadística no paramétrica.

Tabla 3. Estadísticos en la prueba Kruskal Wallis del pan con y sin Bienestarina Mas.

\section{Estadísticos de pruebaa, ${ }^{a, b, c}$}

\begin{tabular}{|l|r|}
\hline & \multicolumn{2}{|c|}{ Medida } \\
\hline Chi-cuadrado & 5,435 \\
GI & 3 \\
Sig. Asintótica &, 143 \\
\hline
\end{tabular}
a. Variable $=$ Fibra
b. Prueba de Kruskal Wallis
c. Variable de agrupación: Sustitución (B) La prueba Kruskal Wallis indica que no se presentaron diferencias significativas entre los cuatro tratamientos ya que en este caso el p-valor es superior al valor de significancia de la prueba $(p=0,143 ; p>$ 0,05). Los valores de fibra dietarían obtenidos, permiten establecer que el pan obtenido pertenece a la categoría de pan integral (NTC 1363). 
Teniendo en cuenta que la recomendación diaria de fibra para niños con edades comprendidas entre 3 y 5 años es de 10 a 13 gramos por cada 1000 calorías (Heimburger et al., (1997) y su recomendación de energía es de 1300 Kcal en promedio, este colectivo requiere 14,95 g de fibra al día, valor que sería cubierto entre un 68 y $72 \%$ por la ración de $40 \mathrm{~g}$ de pan establecidos en la minuta patrón del ICBF 2015.

Tabla 4. Análisis nutricional del pan elaborado con $50 \%$ de sustitución para niños menores de 4 años.

\begin{tabular}{|l|c|}
\hline \multicolumn{2}{|c|}{ Información nutricional } \\
\hline $\begin{array}{l}\text { Tamaño por } \\
\text { porción: }\end{array}$ & $\begin{array}{l}1 \text { unidad }(40 \\
\text { aproximadamente }\end{array}$ \\
\hline \multicolumn{2}{|c|}{ Cantidad por porción } \\
\hline Calorías & $83.74 \mathrm{Kcal}$ \\
\hline Grasa & $1,41 \mathrm{~g}$ \\
\hline Carbohidratos & $12.69 \mathrm{~g}$ \\
\hline Proteína total & $5,05 \mathrm{~g}$ \\
\hline Fibra dietaría & $25,37 \mathrm{~g}(101 \%)$ \\
\hline
\end{tabular}

*Los porcentajes de valores Diarios (VD) están basados en una dieta de 2000 calorías. Sus valores Diarios pueden ser mayores o menores dependiendo de sus necesidades calóricas.

Los resultados obtenidos en la tabla de información nutricional muestran un aporte energético de $83,74 \mathrm{Kcal}$ para $40 \mathrm{~g}$ de pan con sustituciones del $50 \%$ mientras que según estudios de Escobar (1994) se reportan $253 \mathrm{Kcal}$ en una porción de $80 \mathrm{~g}$ aproximadamente $126.5 \mathrm{Kcal}$ en $40 \mathrm{~g}$ lo que indica un menor aporte energético por el producto elaborado con la sustitución del 50 $\%$.

Tabla 5. Comparación nutricional del pan obtenido con 50 \% de sustitución, según la tabla de composición de Alimentos Colombianos y las recomendaciones diarias de consumo de calorías y nutrientes para la población infantil colombiana por el ICBF.

\begin{tabular}{|c|c|c|c|c|}
\hline \multirow[t]{2}{*}{ Nutrientes } & \multicolumn{2}{|c|}{$\begin{array}{c}\text { Pan } \\
\text { tradicional }\end{array}$} & \multirow[t]{2}{*}{$\begin{array}{l}\text { Pan con } \\
\text { sustituc } \\
\text { ión } 50 \%\end{array}$} & \multirow{2}{*}{$\begin{array}{l}\text { Valores } \\
\text { diarios de } \\
\text { referencia } \\
\text { de consumo } \\
\text { de calorinas } \\
\text { y nutrientes } \\
\text { Niños } \\
\text { mayores de } \\
4 \text { años y } \\
\text { adultos }\end{array}$} \\
\hline & $\begin{array}{l}\text { Pan } \\
\text { blandit } \\
0\end{array}$ & $\begin{array}{l}\text { Pan } \\
\text { bla } \\
\text { nco }\end{array}$ & & \\
\hline $\begin{array}{l}\text { Proteína } \\
(\mathrm{g} / 100)\end{array}$ & 8,24 & $\begin{array}{c}6,8 \\
4 \\
\end{array}$ & 12,63 & 50 \\
\hline $\begin{array}{l}\text { Grasa } \\
(\mathrm{g} / 100)\end{array}$ & 6,11 & $\begin{array}{c}3,4 \\
0\end{array}$ & 3,54 & 65 \\
\hline $\begin{array}{l}\text { Fibra Cruda } \\
(g / 100)\end{array}$ & -- & --- & 25,37 & 25 \\
\hline $\begin{array}{l}\text { Carbohidrat } \\
\text { os }(g / 100)\end{array}$ & 52,70 & $\begin{array}{l}68 \\
86 \\
\end{array}$ & 31,74 & 300 \\
\hline $\begin{array}{l}\text { Calorías } \\
\text { (kcal /100) }\end{array}$ & --- & 333 & & 2000 \\
\hline
\end{tabular}

${ }^{*}$ Valores diarios de referencia para el cumplimiento del rotulado nutricional de los alimentos. Fuente: Tabla de composición de alimentos Colombianos. Autor 2014.

De los valores obtenidos se observa que el aporte de proteínas encontrado en el pan con la sustitución del 50 \% es mayor a los panes tradicionales, otro componente importante de destacar es el aporte de fibra el cual sobrepasa el valor diario de referencia. 


\section{CONCLUSIÓN}

La incorporación de Bienestarina Mas $\AA^{\circledR}$ en la elaboración de pan para consumo infantil presentó efectos positivos sobre sus propiedades nutricionales, constituyéndose en una alternativa para garantizar el consumo de este ACF en la población atendida en los programas de asistencia alimentaria y nutricional en Colombia Por medio del ensayo de panificación donde se sustituyó la harina de trigo fortificada por Bienestarina Mas®, se encontró que con la sustitución del $50 \%$ se obtuvo mayor aporte proteico.

\section{REFERENCIAS BIBLIOGRÁFICAS}

Ávila, J. M., Beltrán, B., Cuadrado, C., del Pozo, S., Rodríguez, M. V., Ruiz, E. (2007). La Alimentación Española: características nutricionales de los principales alimentos de nuestra dieta. Ministerio de Agricultura, Pesca y Alimentación.

Escobar, M. 1994. El pan aporte a la buena nutrición. Defensa del alimento. Panadería y pastelería No. 30 Colombia.

Uauy Ricardo, Castillo Carlos (2003). Lipid requirements of infants: implications for nutrient Composition of fortified complementary foods. J Nutr. 133(9):2962S-72S.

UNICEF. (2006). Análisis de situación de los alimentos complementarios fortificados para la niñez entre 6 y 36 meses de edad en la región de América Latina y el caribe.
ICBF. (2014). Distribución, cuidado y uso de un alimento de alto valor nutricional. 2014. Pag 12.

INSTITUTO COLOMBIANO DE NORMAS TECNICAS. (2005). Industrias alimentarias. Pan común, requisitos generales. Bogotá ICONTEC. 132 p. NTC.1363.

Gil Martínez Alfredo. (2010). Pre elaboración y Conservación de Alimentos. Madrid: Ediciones AKAL S.A. (1996). Tratado de Panificación y Bollería. En j. CALAVERAS. España.

Henao Sergio, Johana Aristizabal. (2009). Influencia de la variedad de yuca y nivel de sustitución de harinas compuestas sobre el comportamiento reológico en panificación, Revista Ingeniería e Investigación 29 (1): 39-46. 
Association of the Oficial analytical

Chemists. Official Methods of analysis. (2006). 11th, 12th, 16th y 18 th. Edition. USA. ISBN 0-935584-54-4.

Sanz Neus (2011). Desarrollo de panes especiales con harina integral de amaranto.

Pascual Gloria, Zapata Joaquin. (2010). sustitución parcial de harina de trigo por harina de kiwicha triticum aestivum. amaranthus caudatus I., usando el método directo y esponjay masa, en la elaboración de pan.

Reyes-Aguilar, María José; de Palomo, Patriciay Bressani, Ricardo. (2004). Desarrollo de un producto de panificación apto para el adulto mayor a base de harina de trigo y harina de arroz. Archivos Latinoamericanos de Nutrición. 54(3):314-321.

Heimburger, D. Weinsier, R. (1997). Gastrointestinal and liver diseases. In: Heimburger D, Weinsier R, Butterworth C (Comp.), Handbook of clinical nutrition. (pp. 425 - 444). Philadelphia, Mosby.

Leon Maria Elena, Ydilbrando Misael. (2010). Valor nutritivo de pan con sustitución parcial de harina de trigo (Triticum aestivum) por arracacha (Arracacia xanthorrhiza Bancroft),
Fortificado. Revista venezonala de ciencia y tecnología de los alimentos. 